\title{
Taxonomy of cloud computing services
}

\author{
C.N. Hoefer and G. Karagiannis \\ University of Twente, The Netherlands \\ Email: c.n.hofer@student.utwente.nl,g.karagiannis@utwente.nl
}

\begin{abstract}
Cloud computing is a highly discussed topic, and many big players of the software industry are entering the development of cloud services. Several companies want to explore the possibilities and benefits of cloud computing, but with the amount of cloud computing services increasing quickly, the need for a taxonomy framework rises. This paper describes the available cloud computing services, and proposes a treestructured taxonomy based on their characteristics, to easily classify cloud computing services making it easier to compare them.
\end{abstract}

Index Terms - Characteristics, cloud computing, taxonomy

\section{INTRODUCTION}

Cloud computing (CC) is currently one of the biggest buzzwords and the amount of cloud computing services (CCSs) is increasing rapidly. Many big players of the software industry, such as Microsoft, as well as other Internet technology heavyweights, including Google and Amazon, are joining the development of cloud services [1], [2], [3], [4], [5], [6]. Several businesses, also those not technically oriented, want to explore the possibilities and benefits of CC [7]. However, there is a lack of standardization of CCSs [2], [3], [8], which makes interoperability when working with multiple services or migrating to new services difficult. Further, there is a big marketing hype around $\mathrm{CC}$, where online service providers rebrand their products to be part of the cloud movement [9]. The great amount of different CCSs makes it is hard to compare the offers and to find the right service.

The NIST (National Institute of Standards and Technology) proposed the following definition of cloud computing: "Cloud computing is a model for enabling convenient, on-demand network access to a shared pool of configurable computing resources (e.g., networks, servers, storage, applications, and services) that can be rapidly provisioned and released with minimal management effort or service provider interaction. This cloud model promotes availability." [10]. Further, three service models are currently being differentiated - Software as a Service (SaaS), i.e. online applications, such as web-based email, Platform as a Service (PaaS), which allows customers to deploy their own applications, and Infrastructure as a Service (IaaS), which provides, for example, processing power or storage [10]. However, beyond these categories no further differences are made in current definition.

A cloud can be seen as an infrastructure, see Figure 1, that supports and interconnects several CCSs. The clients, that are the users of the CCSs, are using their home or work computer or any other internet-enabled device to connect and use the CCSs.

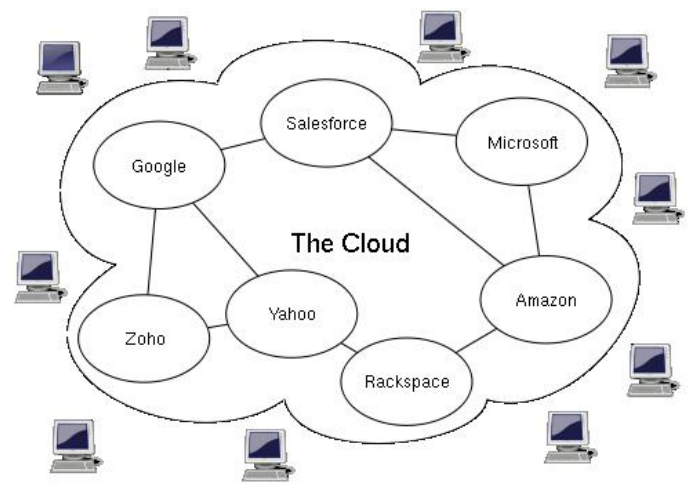

Fig. 1. Cloud computing services [11]

The vast amount of CCSs and the lack of universal definitions and standards leads to the question whether CCSs can be classified in a taxonomy based on their characteristics to easily compare them.

Table-based comparisons of CCSs exist [12], however, they are mainly for commercial use and the degree of detail varies greatly. In [13] a taxonomy has been proposed. However, [13] aims to find the strengths, weaknesses and challenges in current cloud systems, rather than providing a method to compare existing and future CCSs.

In this paper a new, tree-structure based taxonomy is developed, which helps to easily and quickly compare existing and future CCSs. The classification can also help researchers identify areas that could be standardized. The taxonomy will be based on current major cloud computing services, such as the Google App Engine [14], Salesforce.com [15] and Amazon EC2 [16]; but also new developments, such as the Eucalyptus platform [17], will be considered. To create such a taxonomy the following main research question will be answered:

How can cloud computing services be efficiently classified and organized in a taxonomy?

In order to answer this question four sub-questions are proposed, which will provide a step-by-step answer to the main research question.

1) What CCS are currently available?

2) What are the main characteristics that differentiate them?

3) How could the CCS characteristics be used to generate the taxonomy?

4) How can current CCS be categorized in this taxonomy?

This research will mainly be based on literature study, an analysis and comparisons of existing CCSs and the design of the taxonomy. The first two questions are based on literature 
study describing the state of the art. This will provide the information to design the taxonomy, with question one giving an overview of current cloud services in Section 2, and question two addressing the main characteristics of these services in Section 3. Question three is answered in Section 4 , where the design of the taxonomy is described. The forth question will illustrate how CCSs can be compared using the taxonomy and is answered in Section 5. Section 6 concludes and it recommends future activities.

\section{CURREnt CLOUd COMPUTING SERVICES}

The main differences between the CCSs that are deployed are related to the type of service offered, such as (1) storage space and computing power, (2) platforms for own software deployment, or (3) online software applications, ranging from web-email to business analysis tools. Based on these differences, the NIST has already proposed three main categories of CCSs [10]. In this section a few CCSs of each category will be discussed to gain an overview of the existing services.

\section{A. Infrastructure as a service}

Cloud infrastructure services typically offer virtualization platforms, which are an evolution of the virtual private server offerings, that are already known for years [6]. The customers buy the resources, instead of having to set up servers, software and data center space themselves, and get billed based on the resources consumed. They deploy their own software on the virtual machines and control and manage it. The virtual instances can be rented for as long as necessary, which can be as short as an hour. The amount of instances can be scaled dynamically to fulfill the customers needs. Billing is based on this amount, the duration and additional services used, such as additional storage space. Providers often have data centers in multiple locations to offer quick access all over the world. Web interfaces allow monitoring of the cloud service.

Some providers make it possible to connect the virtual instances to the company's network via VPN (Virtual Private Network), to make the company network seem like one big scalable IT infrastructure. These solutions are called hybrid clouds, as they connect the company's (internal) private cloud with the public cloud of the IaaS provider [2].

A pioneer in virtualization and computing power offerings is Amazon [16]. The Amazon Elastic Compute Cloud (Amazon EC2) is one of the most widely used infrastructure platforms [6]. Further popular virtualization services include ServePath's GoGrid [18] and the Rackspace Cloud [19]. Other services are the IBM Smart Business cloud solutions [20], Oracle Cloud Computing [21], GigaSpaces [22], RightScale [23] and Nimbus [24].

Online storage and backup services fall in the category of IaaS. Like most virtualization platform, there are several storage solutions intended for corporate use, but there are also special services for private individuals. Corporate services range from temporal to permanent and from general additional storage space to extend the company's internal capabilities, to storage services aimed at database structured information.
These latter services are billed based not only on the amount of storage space used, but also on the amount of queries on the data. Further, there are specially designed services to extend the storage amount offered with standard virtualization instances.

For private individuals more and more cloud storage and backup services are offered. Laptop and netbook manufacturers, as well as, operating system providers advertise for additional web-storage. Files can be stored on the provider's servers as backup or to synchronize multiple workstations and can often be retrieved from different locations, as the services are often accessible also with a web-browser, such as Rackspace's CloudFiles [19].

Rackspace offers online storage for corporate and private use [19]. Another storage provider is Nirvanix [25]. Amazon offers data storage facilities either in combination or separate from their EC2 instances, called Amazon Elastic Block Store (EBS) and Amazon Simple Storage Service (Amazon S3), respectively [16]. Amazon also provides special database solutions, such as the Amazon SimpleDB [16].

\section{B. Platform as a service}

PaaS providers offer a managed higher-level software infrastructure where customers can build and deploy particular classes of applications and services using the tools and programming languages supported by the provider. The offers include the use of the underlying infrastructure, such as servers, network, storage or operating systems, over which the customers have no control, as it is abstracted away below the platform [10], [6].

Platform services are aimed at specific domains, such as the development of web applications, and are dependent on the programming language. Customers get a separated environment to test and develop or to permanently deploy their applications. Google's App Engine is targeted at traditional web applications offering a Java or Python environment [14]. For small non-scaling applications the Google App Engine is free. On Microsoft's Azure platform applications can be developed using the .NET libraries [1]. Microsoft uses their cloud offers to promote their own software packages [6]. Bungee Connect is specifically designed for cloud application development and deployment [26]. A PaaS of a different domain is Force.com [27], which allows companies to develop customized business applications.

\section{Software as a service}

Cloud software offerings typically provide specific, alreadycreated applications running on a cloud infrastructure. A very well known SaaS is the web-based e-mail. Most software CCS are web-based applications, which can be accessed from various client devices through a thin client interface, such as a web browser. The customers of these services do not manage or control the underlying infrastructure and application platform; only limited user-specific configurations are possible. Features in standard non-remote software applications provid- 
ing internet-based storage are also often considered to be part of SaaS offerings.

A SaaS intended for corporate use is the Salesforce.com service [15], which offers business analysis and customer relationship management (CRM) tools. Appian Anywhere is another domain specific SaaS offering business process management tools [28]. Popular software services also intended for private use are the Google Apps. These include webbased email, calender, contacts and chat capabilities, as well as, the Google Docs package [29], [30], which allows access and sharing of documents, spreadsheets and presentations. Another document sharing and backup service is Box.net [31]. SmugMug is intended for video and photo sharing and uses the Amazon's Simple Storage Service [32].

\section{Open-source based services}

Although some cloud service providers use open-source software or platforms, the base systems are usually proprietary. However, there are a few entirely open-source based platforms, as well as applications and tools available to manage mainly IaaS cloud services. These tools allow the user to monitor, manage and control the virtual instances.

Unfortunately, most open-source CCSs are at the IaaS or PaaS level and very few SaaS open-source applications exist. Further, almost all open-source platforms are based on Linux operating-systems limiting the customer group to these operating-systems [33].

The Eucalyptus cloud is mainly aimed at private clouds [17]. Groundwork is a commercial open-source cloud management system that works with Amazon's EC2 [34]. OpenNebula is a "standard-based open-source toolkit to build private, public and hybrid clouds" [35] and can be used with Amazon EC2. The Nimbus project is also built on an open-source basis. It is maintained by the University of Chicago and was set up for scientific computations [24].

\section{E. New developments}

New developments include offering computer games completely hosted in the cloud. This will make portability easier, as the game can be resumed from a different location [36]. Also it is less dependent on the user's hardware and less prone to piracy.

A rather new and not yet commercially available idea is the offering of $\mathrm{CC}$ resources, such as computing power and data storage, to support smart phones and other resourcestarved devices [37], [38]. Since mobile phones have limited processing power, storage space and battery life, such an offer would make it possible to run more sophisticated applications and offer more services to smart phone users [39].

\section{MAIN CHARACTERISTICS OF CCSS}

As seen above many different CCSs exist. The most apparent difference, the type of service offered, has been addressed. In this section the common characteristics of Iaas, Paas and SaaS cloud services will be examined. Then for each category more specific characteristics will be discussed. It is likely that the list can be expanded further, however, the selected characteristics allow more clear distinctions at each level of the taxonomy.

\section{A. Common characteristics}

The shared characteristics are the license type, the intended user group, the security offered, formal agreements between the provider and the customer, as well as payment systems, interoperability and adherence to standards. In the following sections each of these features will be discussed.

1) License type: Most cloud services use proprietary software and licenses. However, several CC providers make use of open-source software and platforms. Amazon uses the opensource Xen technologies [16] and Google's PaaS offering is built around the open-source Python programming language [14]; but their core cloud computing service and additional services are kept closed-source. A lot of cloud monitoring software is open-source based, as well as, smaller CCSs, since small players often lack the power and influence to push proprietary software on the market [6].

License types also play a role when offering infrastructureand platform-level services. IaaS providers do not suffer from software licensing issues when renting out their virtual servers without operating systems installed. However, when including operating systems and software packages this can cause potential problems as to how the customer should be billed when using the service for a limited time-period. Often additional fees for the software use need to be paid. Other platforms only use their own software, such as Microsoft Azure.

2) Intended user group: Some CCSs differentiate between corporate and private use. Most IaaS and PaaS offerings are intended for companies, whereas SaaS offerings exist for corporations, private individuals or both, such as the Google Apps [30]. However, this does not imply, that services aimed at companies cannot be purchased by individuals.

A further distinction in the corporate and private user group can be made between mobile and fixed users. Mobile users access their CCSs from anywhere, be it at the office, at home, form a desktop, laptop or hand-held. Fixed users are stationary and typically use the same device to connect to the service. Once CCSs intended to support smart phones and other lowresource devices are available (see Section II-E), an additional group, based on this hardware type, can be considered.

3) Security and privacy: Security and privacy are important aspects, especially when important data resides on the cloud's servers. Loss or leak of data can not just cause loss of revenues but also legal actions [4]. In particular, when handling personal data, certain regulations may apply. Due to the absence of standards, cloud security, data privacy and ownership are approached differently by each provider [40].

Generally, encryption and authentication should be used on all cloud services. Encryption can guard, for example, against interception between virtual machines at network level [41]. Due to the low-level of IaaS, the customer has most control over the security compared with PaaS and SaaS. When using 
PaaS the customers may be able to craft their own authentication system or adapt other parts of the system. However, below the application level, security is dealt by the provider, who often gives little or no information about their practices [41]. When using SaaS the user has to rely even more on the provider to implement sufficient security mechanisms.

Most cloud services are accessible with a web-browser and the standard HTTP (Hyper Text Transfer Protocol) is used to connect to the cloud. To provide encryption and secure identification of the server SSL/TLS (Secure Socket Layer / Transport Layer Security) is used. Further security approaches used for authentication and authorization include Public Key Infrastructure (PKI) and X.509 SSL certificates [40]. However, these mechanisms need to be implemented properly. The Amazon EC2 uses public-keys for authentication [41]. For hybrid clouds VPNs are used [2]. The Amazon Virtual Private Cloud (Amazon VPC) service does this [16].

4) Payment systems: The payment system used for CCSs is one of the distinguishing characteristics. The main difference between the traditional form, is that true cloud services are billed based on dynamic use [42], [6]. Rather than paying a fixed monthly or yearly charge, the customer only pays for the resources consumed. The resources could be the number of virtual instances, data storage amount, bandwidth, compute time and resources (CPU or RAM) and transactions (for databases), as well as, combinations of these.

Still cloud computing services can use different payment systems, based on the resources used. The most frequently used pricing model is pay-per-use, in which (resource) units or units per time are associated with fixed price values [42]. When using dynamic or variable pricing, the price is established as a result of dynamic supply and demand, for example, as the means of auctions or negotiations [42]. Zimory.com uses dynamic pricing [43]. A few cloud services are free of charge, such as Google Docs and the Google App Engine (free up to a certain level of computing resources) [14]. Customers of Amazon EC2 are billed monthly for the resources used based on the pay-per-use model [12], [16].

5) Standardization: Standardization refers to the use of technical standards, common APIs (Application Programming Interface) and architectures. These standards can either be approved and "maintained by an organization such as ANSI or the ISO, or they can simply implement a commonly-used or familiar interface (de facto standards)", from [6].

So far there are no defined standards, though this would be beneficial to $\mathrm{CC}$ customers and service developers. Standards would increase interoperability and allow possible customization, due to the technical transparency. Further benefits include price advantages and greater availability of substitutes, because of increased competition. Currently customers are tied to a provider and switching costs are high, due to the incompatibilities of the cloud products [42]. This might be attractive to CC providers, but it implies that customers are subject to price increases, reliability issues or worst, the provider going out of business [1]. Lock-in is one of the biggest obstacles keeping companies from adopting $\mathrm{CC}[6]$. The only way to eliminate this single source failure is to use multiple $\mathrm{CC}$ providers, which is currently hardly possible [1].

There are a several organizations attempting to create such standards [8], including the Cloud Computing Interoperability Forum, which tries to develop a framework that enables two or more clouds to exchange information [44]. Sponsors include IBM, Sun Microsystems, Intel and Cisco. The DMTF's Open Cloud Standards Incubator also aims to standardize interactions between clouds [45]. The Open Cloud Consortium provides testbeds for cloud computing [46]. and the Cloud Standards Wiki tries to gather information about the different organizations working on standards and definitions [47].

6) Formal agreements: The most commonly used formal agreements are service level agreements (SLA), which formally define which level of service the customer can expect and should address latency and QoS (quality of service) [3]. Typically, SLAs include technical specifications of measures, such as uptime or turn around time. Most SLAs also state what compensation the customer can expect in case of failure.

Due to the lack of standards most cloud service providers use SLA agreements to convince potential customers to use clouds "even for mission-critical industrial services, as these SLAs with one single provider are enforceable", from [42].

\section{B. Specific characteristics}

The most important characteristics of CCSs are explained above. As for the common characteristics, more specific features of infrastructure, platform and software cloud services often do not allow clear distinctions within the chosen characteristic, therefore only a few features are discussed below.

1) IaaS-specific characteristics: A characteristics to consider are the supported operating systems and applications/frameworks, as this might be important to potential customers. Most IaaS providers support Linux systems, but some also have Windows and OpenSolaris support. Widely supported applications include the MySQL database and the Apache HTTP Server software. Another characteristic that is important for developers is whether and what kind of development tools the provider supplies. This could include an API or special command-line tools [12]. Services comprising virtual instances can be further differentiated based on the virtualization technology used. Xen [48] is currently used by most providers [12].

2) PaaS-specific characteristics: An important PaaS characteristic is related to which programming languages and platforms are supported. Google's App Engine, for example, currently only supports Python and Java environments. The supported operating systems and applications can also be a relevant feature.

3) SaaS-specific characteristics: Software cloud services vary a lot. A characteristics to consider is the customer/application domain of the offered service. This domain could be customer relations or other business management areas, office applications, social networking, and data exchange. 


\section{TAXONOMY DESIGN}

Having analyzed the important features of CCSs, the different levels have to be defined and the taxonomy can be generated. Then a brief explanation of the choice of levels is given.

The taxonomy has a tree-based structure. At the root of the tree are all cloud services. The first level is made up of the three main service categories, see Section III. The next levels correspond to the common characteristics, followed by the service specific characteristics. The taxonomy levels are:

1) Main service category

2) License type

3) Intended user group

4) Payment system

5) Formal agreements

6) Security measures

7) Standardization efforts

The IaaS levels are:

a. Supported operating systems

b. Supported applications and frameworks

c. Available development tools

d. Virtualization technology

The PaaS levels are:

a. Supported languages and environments

b. Supported operating systems

c. Supported applications and frameworks

The SaaS levels are:

a. Customer/application domain

The tree traversal starts at the top at level 0 . There a choice needs to be made based on the first level's characteristic. Further down in the tree it can be possible that multiple choices can be made, for example, when considering which operating systems are supported.

The license type and intended user group have been chosen as a very important criteria because decisions made at these levels will lead to very different cloud services. The remaining order was chosen based on the amount of information found on the characteristics, i.e. the more information was found, the higher up in the tree a characteristic is placed. However, general and service specific characteristics are kept separated. At deeper levels the distinctions between services may become more blurred, as either little information is available, or implementations only vary slightly. Here, it may also be applicable to add tree nodes labeled 'Undisclosed/unknown' when sufficient information is not available.

The taxonomy examined the main features of CCSs. The level of detail can be varied by including more options for each characteristics. The taxonomy can also be expanded with additional characteristics, i.e. tree levels, such as interoperability with other providers or how well the to be classified CCS can be customized to the user's needs. Further, the security characteristic currently only considers those measures taken to secure the connection from a client to the cloud. The security and privacy mechanisms used within the cloud, are left out.

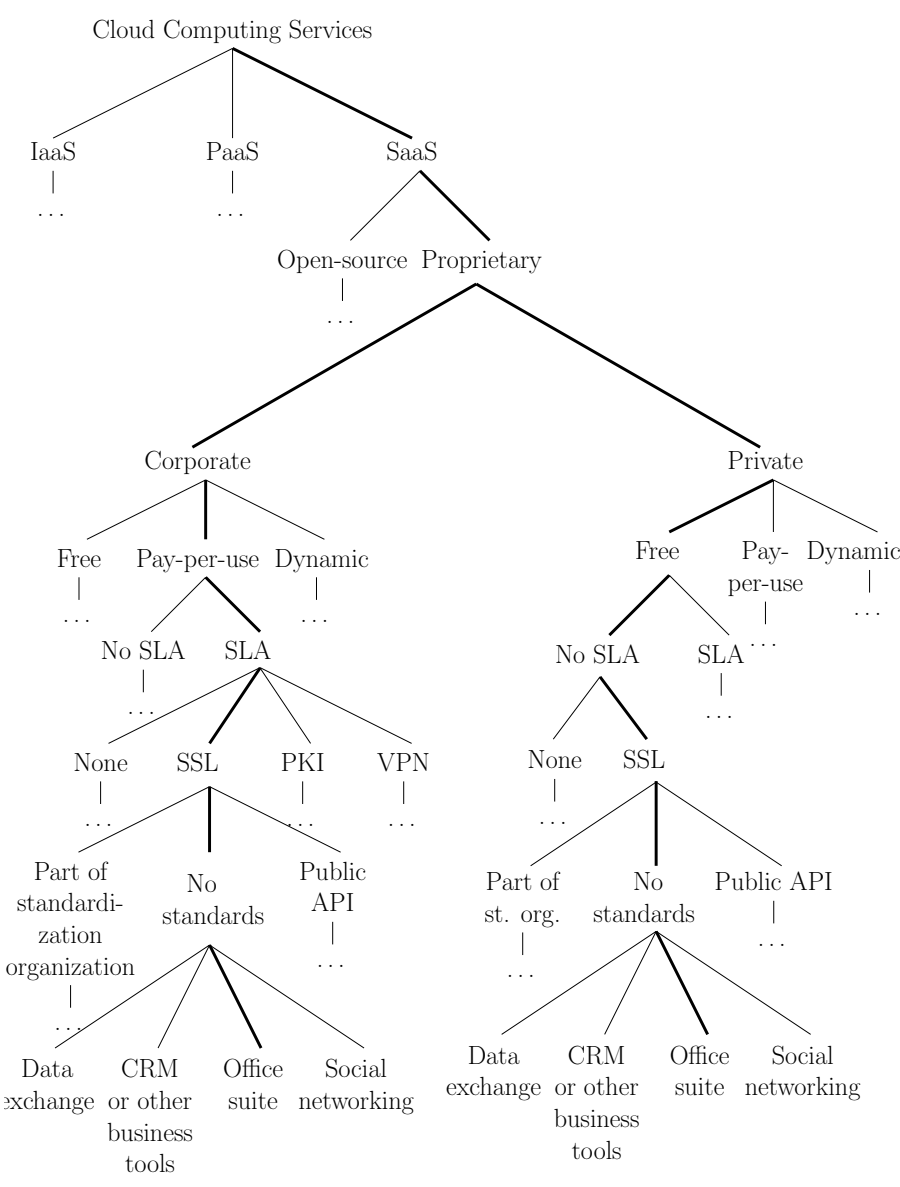

Fig. 2. Google Apps Tree

These could concern, securing the traffic between a customer's virtual instances, or separating multiple customers' data.

\section{Classifying CCSS IN THE TAXONOMY}

This section explains with an example how the taxonomy is to be used. The characteristics of the Google Apps [30], [29] are given in Table I. The corresponding tree diagram can be found in figure 2. The Google Apps characteristics define the tree path indicated by the bold line.

TABLE I

GOOGLE APPS CHARACTERISTICS

\begin{tabular}{|l|l|}
\hline Level & Found characteristics \\
\hline 1. Service & SaaS \\
\hline 2. License & Proprietary \\
\hline 3. User group & Corporate and private use \\
\hline 4. Payment & $\begin{array}{l}\text { Free for personal use (Standard Edition), 50\$ per } \\
\text { account per year for business use (Premier Edition) }\end{array}$ \\
\hline 5. Agreements & No SLA for Standard Ed., with SLA for Premier Ed. \\
\hline 6. Security & HTTPS \\
\hline 7. Standards & No standards, (Single sign-on API for Premier Edition) \\
\hline a. Domain & Office suite (incl. email, calender, collaboration tools) \\
\hline
\end{tabular}

\section{CONCLUSIONS AND FUTURE WORK}

This paper examined current cloud computing services (CCSs) and a taxonomy for classifying these has been presented. The paper captured the characteristics all CCSs share, 
as well as those that are exclusive to one of the three service categories - infrastructure, platform and software.

The here proposed taxonomy is capable of classifying both current and future CCSs. The simple tree structure allows quick comparisons, by giving the user a set of choices at each level. This clear structure makes comparing CCSs more efficient than using table based comparisons. Further, the taxonomy not only helps to map a CCS, but it also helps potential customers and developers to point out what characteristics the service they seek or wish to develop should have.

The comprehensive list of characteristics makes it possible to distinguish a great variety of CCSs. However, at the deeper levels of the tree, the differences between the characteristics become more blurred. This may be due to limited subjective information, and the young age of cloud computing. To allow more accurate comparisons the taxonomy could be expanded to incorporate more details for some of the characteristics. As mentioned above, the security addressed by the taxonomy only considers security measures between the client and the cloud, but not those security mechanisms used within the cloud. This would be an important addition to the taxonomy.

Further, it has been identified that especially the areas of standardization and interoperability need to evolve. Various organizations have been founded to help define concepts and standards for cloud computing, but also the service providers need to be convinced to take part. Improved interoperability and clear standards will not only make it easier to develop new cloud services, but it will also make entering the cloud less risky, and hence more attractive, for companies. The taxonomy can easily be adapted to future cloud computing developments and the taxonomy's user's needs.

\section{REFERENCES}

[1] M. Armbrust, A. Fox, R. Griffith, A. D. Joseph, R. H. Katz, A. Konwinski, G. Lee, D. A. Patterson, A. Rabkin, I. Stoica, and M. Zaharia, "Above the Clouds: A Berkeley View of Cloud Computing," EECS Dept., Uni. of California, Berkeley, Tech. Rep. UCB/EECS-2009-28, Feb '09.

[2] L. Qian, Z. Luo, Y. Du, and L. Guo, "Cloud computing: An overview," in CloudCom '09: Proceedings of the 1st International Conference on Cloud Computing. Springer-Verlag, 2009, pp. 626-631.

[3] G. Lin, D. Fu, J. Zhu, and G. Dasmalchi, "Cloud computing: It as a service," IT Professional, vol. 11, no. 2, pp. 10-13, 2009.

[4] P. Murray, "Enterprise grade cloud computing," in WDDM '09: Proceedings of the Third Workshop on Dependable Distributed Data Management. New York, NY, USA: ACM, 2009, pp. 1-1.

[5] A. Weiss, "Computing in the clouds," NetWorker, vol. 11, no. 4, pp. 16-25, 2007.

[6] D. Hilley, "Cloud computing: A taxonomy of platform and infrastructure-level offerings," CERCS, Georgia Institute of Technology, Tech. Rep. GIT-CERCS-09-13, April 2009.

[7] T. Jowitt, "Four out of five enterprises giving cloud a try," August 27, 2009. Computerworld UK, (visited: 2010, May 7). [Online]. Available: http://www.computerworlduk.com/management/ it-business/services-sourcing/news/index.cfm?newsId=16355

[8] R. Grossman, "The case for cloud computing," IT Professional, vol. 11, no. 2, pp. $23-27$, march-april 2009.

[9] A. Plesser, "CC is hyped and overblown, Forrester's Frank Gillett.....Big tech companies have 'cloud envy',' Sep '08, interview video (visited: 2010, Apr 23). [Online]. Available: http://www.beet.tv/2008/ 09/cloud-computing.html

[10] P. Mell and T. Grance, "The NIST definition of cloud computing (v15)," National Institute of Standards and Technology, Tech. Rep., 2009.
[11] S. Johnston, "Diagram showing overview of cloud computing including Google, Salesforce, Amazon, Microsoft, Yahoo \& Zoho," (visited: 2010, May 23). [Online]. Available: http://en.wikipedia.org/wiki/File: Cloud_computing.svg

[12] Tippit Inc., WebHostingUnleashed, "Cloud-computing services comparison guide," 2008, (visited: 2010, Feb 3). [Online]. Available: http://www.itsj.com/Resources/cloud-computing-comparison.pdf

[13] B. P. Rimal, E. Choi, and I. Lumb, "A taxonomy and survey of cloud computing systems," in NCM '09: Proceedings of the 2009 Fifth International Joint Conference on INC, IMS and IDC. Washington, DC, USA: IEEE Comp. Society, 2009, pp. 44-51.

[14] Google App Engine. [Online]. Available: http://appengine.google.com

[15] Salesforce.com. [Online]. Available: http://salesforce.com

[16] Amazon Web Services. [Online]. Available: http://aws.amazon.com

[17] Eucalyptus. [Online]. Available: http://open.eucalyptus.com

[18] GoGrid. [Online]. Available: http://www.gogrid.com

[19] Rackspace Cloud. [Online]. Available: http://www.rackspacecloud.com

[20] IBM Cloud Computing. [Online]. Available: http://ibm.com/ibm/cloud

[21] Oracle Cloud Computing. [Online]. Available: http://oracle.com/us/ technologies/cloud

[22] GigaSpaces.com. [Online]. Available: http://www.gigaspaces.com

[23] RightScale. [Online]. Available: http://www.rightscale.com

[24] University of Chicago, "Nimbus is cloud computing for science." [Online]. Available: http://www.nimbusproject.org

[25] Nirvanix. [Online]. Available: http://www.nirvanix.com

[26] Bungee Labs. [Online]. Available: http://www.bungeeconnect.com

[27] Force.com. [Online]. Available: http://force.com

[28] Appian Anywhere. [Online]. Available: http://www.appian.com/ bpm-saas.jsp

[29] D. R. Herrick, "Google this!: using Google Apps for collaboration and productivity," in SIGUCCS '09: Proceedings of the ACM SIGUCCS fall conference on User services conference. New York, NY, USA: ACM, 2009, pp. 55-64.

[30] Google Apps. [Online]. Available: http://www.google.com/apps

[31] Box.net. [Online]. Available: http://box.net

[32] SmugMug. [Online]. Available: http://www.smugmug.com

[33] T. Xia, Z. Li, and N. Yu, "Research on cloud computing based on deep analysis to typical platforms," in CloudCom '09: Proceedings of the 1st International Conference on Cloud Computing. Berlin, Heidelberg: Springer-Verlag, 2009, pp. 601-608.

[34] GroundWork. [Online]. Available: http://www.groundworkopensource. com

[35] OpenNebula Project. [Online]. Available: http://www.opennebula.org

[36] C. Edwards, "The Tech Beat: Games in the Cloud?" Feb. 24, 2009. Business Week, (visited: 2010, Apr 28). [Online]. Available: http://www.businessweek.com/the_thread/techbeatarchives/ 2009/02/games_in_the_cl.html

[37] ABI Research, "Mobile Cloud Computing - Next Generation Browsers, Widgets, SIM, Network-as-a-Service, and Platform-asa-Service," (visited: 2010, May 31). [Online]. Available: http: //www.abiresearch.com/research/1003385-Mobile+Cloud+Computing

[38] Intel Labs Berkeley, "Clone Cloud," (visited: 2010, May 31). [Online]. Available: http://berkeley.intel-research.net/bgchun/clonecloud

[39] C. Mims, "Technology Review: Sending Cell Phones into the Cloud," May 2009, (visited: 2010, May 31). [Online]. Available: http://www.technologyreview.com/communications/22571/

[40] L. Youseff, M. Butrico, and D. Da Silva, "Toward a unified ontology of cloud computing," in Grid Computing Environments Workshop, 2008. GCE '08, Nov 2008, pp. 1-10.

[41] J. Viega, "Cloud computing and the common man," Computer, vol. 42, pp. 106-108, 2009.

[42] C. Weinhardt, A. Anandasivam, B. Blau, N. Borissov, T. Meinl, W. Michalk, and J. Stößer, "Cloud computing-a classification, business models, and research directions," Business and Information Systems Engineering (BISE), vol. 1, no. 5, pp. 391-399, 2009.

[43] Zimory GmbH, "Building the flexible data center." [Online]. Available: http://zimory.com

[44] CCIF. [Online]. Available: http://cloudforum.org

[45] DMTF Cloud Computing Incubator. [Online]. Available: http://www. dmtf.org/about/cloud-incubator

[46] Open Cloud Consortium. [Online]. Available: http:// opencloudconsortium.org

[47] Cloud-Standards.org. [Online]. Available: http://cloud-standards.org

[48] Citrix Systems, Inc., "Xen hypervisor." [Online]. Available: http: //www.xen.org 